\title{
¿Hay que eliminar el sistema binominal? Una propuesta alternativa ${ }^{1}$
}

J. Samuel VALENZUELA

$\mathrm{E}$ 1 sistema binominal mayoritario por lista que se usa para las elecciones parlamentarias chilenas constituye una adición peculiar y única a la gran variedad de fórmulas electorales que registra la historia de las democracias. Como es bien sabido, su origen es de dudosa legitimidad. Formó parte de las llamadas "leyes de amarre" que la dictadura de Augusto Pinochet produjo después de la realización del plebiscito de julio de 1989, el cual aprobó una serie de reformas democratizantes a la Constitución de 1980. Si bien este plebiscito representó la culminación de una rueda de negociaciones con la oposición de la época, la ley electoral fue preparada en secreto solamente por los asesores de la dictadura e impuesta sin consulta alguna. No cabe duda que su motivación fue la de favorecer a la segunda lista ganadora en las elecciones. Como se sabía que las fuerzas unidas en la Concertación de Partidos por el No, que lograron derrotar a Pinochet en el plebiscito de octubre de 1988, obtendrían la mayoría de los votos pero no alcanzarían a los dos tercios de la votación salvo en contados lugares, la idea matriz fue desarrollar un mecanismo que le diera el $50 \%$ de la representación parlamentaria a la lista que obtuviera al menos un tercio de los votos.

1. Desarrollé las ideas expuestas en este artículo originalmente para un seminario sobre "Las propuestas de reformas constitucionales al sistema electoral mayoritario binominal", que fue organizado a fines de noviembre de 2003 por Bernardo Navarrete en el Instituto de Asuntos Públicos del Departamento de Ciencia Política de la Universidad de Chile. Mis agradecimientos a Bernardo Navarrete, Ricardo Wilhem, Miguel Ángel López y René Cortázar por sus observaciones a ellas. 
Según la inusual fórmula electoral concebida con esa motivación, los partidos deben presentar un máximo de dos candidatos por unidad territorial de representación. Realizada la elección, se suman los votos de las listas presentadas en cada distrito o circunscripción, y la que obtiene más votos elige a los dos candidatos que presentó, pero sólo siempre y cuando doble el número de votos recibidos por la lista que le siga en votación. De lo contrario, las dos primeras listas ganadoras eligen al candidato o candidata más votado entre sus dos opciones. Al haber dos listas, basta que la segunda obtenga una fracción más que un tercio para elegir a un representante. $\mathrm{Al}$ haber más listas, este umbral de representación obviamente disminuye en función de cuántos votos reciban las posibles terceras, cuartas, o ene listas.

El sistema binominal tiene numerosos defectos que perjudican sobre todo a la Concertación democrática, la coalición sucesora a la que se agrupó en torno al "no", por la sencilla razón que tiene más partidos que la Alianza por Chile, la coalición formada por los dos partidos de derecha. Por ello y dado el origen básicamente ilegítimo del sistema (¿qué democracia funciona con un sistema electoral diseñado por una dictadura para favorecer a sus partidarios?), no han faltado desde el retorno a la democracia las voces que claman por cambiarlo.

Pero el sistema binominal tiene un efecto importante: obliga a los partidos afines a aglutinarse en dos grandes coaliciones. Las terceras listas electorales no eligen a nadie, y si son formadas por un partido o partidos con votaciones importantes, lo único que hacen es aumentar las probabilidades de que la primera lista ganadora doble los votos de la segunda. Cualquier líder político racional sabe, en consecuencia, que no hay otra alternativa más que la de formar la coalición más amplia posible entre partidos que cuenten con la afinidad mínima necesaria para hacerlo. En consecuencia, la organización actual del sistema partidario chileno no es solamente el producto de una inercia política que se alarga desde el "sí" y el "no" en el plebiscito que derrotó a Pinochet. Es también el producto de este sistema electoral que opera como una camisa de fuerza transformando el pluripartidismo chileno en un sistema que se organiza en torno a dos grandes coaliciones. Con otro sistema electoral, las disputas que ha habido entre los componentes de ambas coaliciones (de especial acrimonia en la derecha) ya hubieran roto la estructuración coalicional del sistema partidario.

Desde el retorno a la democracia no han faltado voces que clamen por cambiar el sistema electoral ni los proyectos de reforma para hacerlo. Nada ha pasado, sin embargo, dada principalmente la oposición de los partidos de derecha. Todas las propuestas de cambio han tenido como supuesto básico de que hay que volver a un sistema proporcional, preferentemente a uno que establezca algún nivel mínimo de votación nacional para poder participar en la repartición proporcional de escaños. Sin embargo, dicho nivel mínimo no podría ser muy alto, y por lo mismo no impediría el riesgo de que el sistema partidario chileno vuelva a fraccio- 
narse como sucedió en el pasado. Además, aun si no se fraccionase mayormente, de todas maneras se perdería el efecto aglutinante que tiene el sistema binominal sobre el pluripartidismo chileno. La organización en dos coaliciones ha facilitado la gobernabilidad del país en estos años, y con una vuelta a la proporcionalidad -especialmente a estas alturas en que se ha debilitado la división político-electoral que se originó en el "sí" versus "no" - dicha organización bien puede quedar relegada a la historia de las transformaciones del sistema partidario chileno ${ }^{2}$. El gobierno de Gabriel González Videla (1946-1952), con sus coaliciones siempre cambiantes, ilustra bien lo que podría suceder con la gobernabilidad del país.

En lo que sigue, propongo una alternativa de reforma al sistema binominal que reduce o elimina sus defectos, pero no altera el aspecto fundamental que genera la estructuración en dos grandes coaliciones: el hecho que se sumen los votos por lista para ver si hay doblaje o no antes de determinar quiénes resulten elegidos. Al exponer cómo funcionaría esta alternativa mencionaré cómo operaría para neutralizar los defectos del sistema actual. En esencia, lo que propongo es instituir un segundo cómputo de los votos que llamaré "remanentes", es decir aquéllos que fueron expresados claramente pero no sirvieron para elegir a nadie. Pero antes, es necesaria otra reforma.

\section{Abrir los cupos}

El sistema binominal tiene un aspecto que no es esencial a su funcionamiento. Consiste en el hecho de que las listas de candidatos que se inscriben antes de la elección en el Servicio Electoral tengan, necesariamente, que limitarse a un máximo de dos por unidad territorial de representación. Si bien en un sistema mayoritario simple y de una vuelta es necesario que cada lista presente a un solo candidato para que funcione bien -el uninominalismo le es prácticamente intrínseco y el electorado vota directamente por una persona y no un partido-, bajo el binominalismo mayoritario por lista, que es más bien un sistema proporcional que uno mayoritario, no es necesario que las listas se limiten a dos candidatos solamente porque se eligen a dos representantes por distrito o circunscripción. A pesar de que los electores estén votando actualmente en Chile por una persona, los votos se suman primero por lista, con lo cual en el fondo los votos son más bien para la lista que para las personas, y en no pocos casos "ganan" la elección candidatos que reciben menos votos que sus oponentes en la lista contraria ${ }^{3}$. En este sentido, el

\footnotetext{
2. Para un análisis de las transformaciones del sistema partidario chileno, véase J. Samuel Valenzuela (1995), pp. 5-77.

3. Es lo que le sucedió, por ejemplo, a Andrés Zaldívar en la elección de 2005: obtuvo unos 284.800 votos pero perdió frente a Jovino Novoa que consiguió 256.300. Un ejemplo más dramático, dadas las cifras respectivas, ocurrió en la elección senatorial de 1989. Jaime Guzmán obtuvo 224.302 votos, pero fue elegido sobre Ricardo Lagos, quien obtuvo 407.890 votos.
} 
sistema mayoritario simple de una vuelta es la excepción; todos los demás sistemas funcionan igual de bien, o mal, con más candidatos que puestos por llenar. Sería distinto si el sistema electoral chileno fuera binominal mayoritario por persona, y no por lista, ganando la elección quienes saquen individualmente la primera y segunda mayorías de votos en cada lugar. Pero si los asesores de la dictadura hubieran estructurado así la fórmula electoral, no habría habido ninguna garantía que la segunda lista ganadora obtuviese la mitad de la representación con algo más de un tercio de los votos (al haber dos listas), y habría quedado planteada la pregunta: ¿por qué no instituir en ese caso unidades de representación uninominales eligiendo a quienes obtengan la primera mayoría simple?

No lo hicieron así, de modo que nada impida que se instituya en el sistema binominal chileno la primera reforma que sugiero, que consiste en eliminar la limitación a dos candidatos por lista y por unidad de representación. Esta limitación sólo genera defectos que entorpecen el funcionamiento de la democracia. Las consecuencias negativas se visualizan sobre todo en la Concertación. Esta regla díscola impide que todos los partidos que componen la Concertación presenten candidatos en cada distrito o circunscripción. Con ello se desactivan y desaniman las bases locales de los partidos que no pueden trabajar por un candidato de sus filas, y los electores que preferirían a candidatos de tales partidos se ven obligados a votar por su segunda opción. Además, las bases locales de los partidos que quedan sin un candidato de sus tiendas, y sus electores, suelen dividirse a la hora de decidir a quién van a apoyar, lo que genera un sinnúmero de problemas. Una democracia madura no puede funcionar bien si su sistema electoral produce efectos contraproducentes sobre la vida y viabilidad de sus partidos.

La estrechez de los cupos impide, además, que sepamos a ciencia cierta cuál es el apoyo real que tienen los partidos nacionalmente en las elecciones legislativas. ¿Cómo vamos a saber qué votación tiene el Partido Radical Social Demócrata, por ejemplo, en comparación con el Partido Comunista, si los candidatos del primero aparecen en tan pocos distritos? Hay que recurrir a las elecciones municipales para tener una estimación de este apoyo, pero estas elecciones no son las más convenientes para ello por razones que huelga repetir.

Y el hecho de que los partidos no puedan llevar todos sus propios candidatos en cada unidad lleva a la "cocina política" de los cupos. Los partidos de menor electorado relativo tienen que asegurar que se les dé la posibilidad de presentar sus candidatos más importantes en los lugares donde más chances tienen, para lo cual les conviene que sus partidos aliados los pareen allí con candidatos más débiles. Y así sucesivamente. La elección parlamentaria no depende de este modo solamente de la voluntad de los electores, sino que también -y esto a un grado que excede lo que ocurre en otros sistemas electorales- de la manera en que los dirigentes políticos confeccionan las listas. 
Al permitir que cada coalición de partidos presente los candidatos que quiera -hasta un máximo de cuatro o cinco- en cada unidad de representación, se eliminan estos problemas. Las bases locales de cada partido podrán trabajar para su candidato, y los electores podrán todos votar por su primera opción. Las elecciones para la Cámara de Diputados volverán así a ser la principal medida de la fuerza de los partidos a nivel nacional. Las discusiones sobre qué candidatos se presentarán en cada lugar volverán a radicar principalmente donde corresponde que se den, es decir, en los partidos mismos, y las coaliciones no se pasarán largas horas negociando los cupos antes de inscribir sus listas de candidatos en el Servicio Electoral. Y si bien el incremento de los cupos tendrá el efecto de aumentar potencialmente el conflicto, que ya se produce entre los candidatos de la misma lista en los lugares donde tienen pocas probabilidades de doblar a sus contrincantes de la otra lista principal, de todas maneras todos los candidatos y sus electores sabrán, dada la segunda reforma que propongo, que los votos que reciban servirán para otro cómputo en caso que pierdan. Con éste, la elección en cada distrito o circunscripción perderá algo de su aspecto de juego suma/cero, como ocurre actualmente, lo que beneficiará las relaciones internas en cada coalición. Los partidos de los candidatos perdedores no "perderán" completamente los votos que éstos recibieron, y con ello, por lo mismo, cada colectividad tendrá interés en presentar sus mejores opciones en cada lugar. Se acaba así la programación de las listas con la inclusión deliberada de candidatos débiles destinados a contribuir a la victoria de sus compañeros de lista. Todo esto quedará más claro con la discusión del que llamo "voto remanente" más abajo.

A primera vista, la ampliación de los cupos no conviene a los partidos de menor votación nacional ya que su representación parlamentaria, a diferencia de los partidos más grandes, depende tanto o más de las negociaciones al conformar las listas de candidatos que de la voluntad de los electores. Pero los partidos más chicos a menudo tienen su voto concentrado en algunos lugares del país (lo cual vale incluso para el que menos votos tiene en las dos grandes coaliciones, es decir, el PRSD), de modo que aún pueden aspirar a obtener escaños en ellos. En todo caso, no cabe duda que al haber más cupos de candidatos y una fragmentación mayor de los votos de la coalición entre ellos, esto podría redundar en cierta ventaja para los partidos de mayor apoyo electoral. En consecuencia, una reforma que aumente el número de cupos de candidatos podría estimular paradójicamente la fusión de los partidos más chicos. Este incentivo a la fusión será fortalecido levemente con la segunda reforma que propongo en torno al voto remanente. Obviamente, este efecto que apoyaría la mantención de un pluripartidismo muy moderado en vez de extremo en el sistema partidario chileno no se daría al adoptarse un mecanismo plenamente proporcional. 


\section{Los "votos remanentes" y su cómputo}

Naturalmente, al conservarse el mecanismo básico del sistema binominal seguirá dándose el hecho de que algunos electores de cada coalición contribuirán con sus votos a elegir representantes de otros partidos de la coalición. En todo caso, con el segundo cómputo este efecto del sistema binominal se aminorará ya que con él todas las preferencias de la ciudadanía podrán favorecer la elección de algún candidato o candidata del partido que han votado.

Los "votos remanentes" de cada partido se componen de dos fuentes. La primera son las votaciones extraordinariamente altas que suelen recibir algunos candidatos que resultan elegidos. Para determinar el número de estos votos que pasarían al cómputo de los votos remanentes, se debe dividir el total de votos efectivos en la elección por el número de vacantes por llenar. Esto genera el promedio nacional de votos efectivos para elegir un senador o diputado. Así, en la votación de diputados de 2005 hay que dividir 6.518.001 por 120, lo cual da un cuociente de 54.316 . La porción de los votos individuales de los candidatos electos que excedan este número promedio constituye la primera fuente para conformar el voto remanente.

Hay pocos candidatos que exceden el promedio de votos por vacante dado el gran número de candidatos y la distribución de muchos de ellos en distritos poco poblados. Es hacer justicia al apoyo que estos votos representan para la votación nacional de los partidos respectivos el pasar la proporción excedentaria del promedio nacional al voto remanente. Estos votos provienen, además, de distritos y circunscripciones más poblados (sólo así pueden exceder los promedios nacionales), y el hecho de que cuenten para el segundo cómputo ayuda a corregir otro gran defecto del sistema electoral chileno: el hecho que los distritos y circunscripciones tienen niveles poblacionales tan desiguales, produciendo un déficit de representación de los lugares más poblados.

La segunda fuente de los votos remanentes, la más importante en términos numéricos, estaría constituida por todos los votos expresados por aquellos candidatos de cada partido que no hayan sido derrotados por doblaje, pero que igual no sirvieron para elegir a los candidatos respectivos. En otras palabras, hay que sumar los votos de los candidatos perdedores de cada localidad en que resultó elegido un solo candidato de cada lista ganadora. Como ya mencioné, estos votos exceden a veces los que obtuvieron individualmente los candidatos de la lista contraria que fueron, sin embargo, elegidos ${ }^{5}$. La suma de estos votos debe hacerse por partido (o grupo "independiente" con afinidades claras entre sí), sin poder emparentarse las listas partidarias para efectos de esta suma. De lo contrario,

4. En todas las cifras referidas a la elección de 2005, uso las que están disponibles a sólo días de la elección en el sitio web elecciones.gov.cl No son, por lo tanto, cifras finales oficiales, sino las que da a horas de la elección el Ministerio del Interior.

5. Véase la acotación 2. 
se estarían transfiriendo las preferencias claramente marcadas por los electores por un partido equis a otro, operación que violaría el propósito y los efectos benéficos sobre el sistema de partidos del voto remanente. La idea es que el voto remanente sirva para consolidar el acercamiento entre las opciones ciudadanas y la oferta partidaria, y no para ofuscarlo. Esta regla previene además el fraccionamiento partidario.

Al sumar los votos remanentes, se deben descartar todos los que fueron expresados por candidatos que perdieron frente a una lista que consiguió doblar a la segunda. En estos casos queda claro que ambos candidatos de la segunda lista más votada fueron derrotados. No hay que retener tampoco los votos de las listas adicionales en estas situaciones. Sería absurdo agregar los votos de las terceras o cuartas listas pero descartar los que obtuvieron los candidatos que perdieron en la segunda ${ }^{6}$.

El segundo cómputo con los votos remanentes determinaría quiénes serían electos para ocupar un cierto número fijo de escaños que habría que agregar a los existentes. Tanto la Cámara como el Senado tienen actualmente menos miembros que los que había antes de 1973, y no hay ninguna razón por la cual no se pueda expandir su número. En la Cámara estos escaños adicionales podrían ser quince, y en el Senado seis (agregando tres al total en cada renovación parcial), aunque estos números podrían ser algo mayores. Sin embargo, no conviene ampliarlos mucho más, ya que de hacerlo, el segundo cómputo dejaría de incentivar un pluripartidismo moderado y podría comenzar a premiar la representación (aunque sea de una sola persona) de una gama demasiado amplia de partidos pequeños, estimulando un empresarismo político.

Para asignar los nuevos escaños con los votos remanentes propongo recurrir a una proporcionalidad simple. Luego de calcular un cuociente que resultaría de dividir el total de dichos votos por el número de escaños por llenar (éste viene a ser el cuociente llamado "de Hare" en la literatura sobre sistemas electorales), habría que dividir la votación remanente de cada partido por dicha cifra ${ }^{7}$. Esta operación debe incluir la votación recibida por los partidos que no forman parte de las dos coaliciones principales, cuyo voto "remanente" normalmente será igual a su votación total por tener una bajísima probabilidad de elegir a alguien en la fase binominal mayoritaria por lista de los cómputos. La división con el cuociente de Hare permite asignar una primera ronda de escaños a los partidos cuyo voto remanente lo excede. Una vez hecha esta determina-

\footnotetext{
6. Una vez calculado el voto remanente, se puede generar, para efectos del análisis de la elección, un "índice de efectividad de la votación" por partido. El índice resulta de la división del voto remanente por la votación total recibida por el partido, restándose luego el porcentaje resultante de 100 o de 1 (si no se calcula el valor recíproco, el índice viene a ser de la "inefectividad de la votación"). Este índice muestra la poca efectividad de la votación democratacristiana en las elecciones de diputados de 2005, ya que apenas alcan$\mathrm{za}$ al $48,5 \%$. Así, menos de la mitad de sus votos sirvieron para elegir a alguien de su partido. En cambio el mismo índice para el PRSD es de 91,6\%, y el de la UDI de 68,6\%.

7. Este procedimiento está explicado en David M. Farell (1998), pp. 62-63.
} 
ción, hay que transferir los montos que restan para cada partido (a menudo significarán la totalidad de su votación) a una nueva lista de votos remanentes. Los escaños restantes se deben asignar hasta completar su número a los partidos en orden descendente, según el tamaño de su votación remanente. Y dichos escaños deben asignarse a los candidatos que obtuvieron la mayor votación en cada partido entre quienes no ganaron con el cómputo binominal. De este modo, resultarán elegidos un número mayor de representantes de distritos o circunscripciones más poblados, ayudando nuevamente a corregir las desproporciones actuales en este aspecto del sistema electoral chileno. En todo caso, los candidatos así elegidos serán también los que más habrán aportado, en el conjunto de quienes no fueron favorecidos por el mecanismo binominal, a la votación remanente de sus partidos respectivos.

Naturalmente, la operación de este sistema será distinta y más completa con elecciones en las cuales se hayan presentado todos los partidos que componen el espectro partidario chileno en cada, o en la mayoría, de los distritos y circunscripciones del país. No es así actualmente, ya que para ello se necesita primero la institución de la primera reforma que propongo aquí. En todo caso, conviene ejemplificar el segundo cómputo que sugiero con las elecciones parlamentarias de diciembre de 2005 para visualizar mejor su impacto.

La elección de diputados produjo 2.121.345 votos remanentes, de los cuales solamente 46.452 provinieron de los votos excedentarios al promedio de la votación nacional por diputado. Estos surgieron de la votación recibida por cinco diputados electos: dos del PS, y los tres restantes de RN, del PDC y del PPD, respectivamente. Si suponemos que se completan sólo 15 escaños adicionales, el coeficiente de Hare viene a ser de 141.423. En el Cuadro 1 se puede examinar la operacionalización de la distribución de los escaños por partidos.

Como puede observarse, el PDC adiciona cinco diputados con este cómputo, la UDI a tres, RN y el PC a dos, y el PPD, el PS y el PH a uno. El segundo cómputo permite así una representación parlamentaria de partidos que no forman parte de las dos coaliciones principales, es decir, de los partidos Comunista y Humanista. Esta reforma subsana, por lo tanto, otro defecto del sistema binominal mayoritario: el hecho que tiene un efecto tan excluyente de los partidos que se agrupan en coaliciones más chicas, que ello les cierra casi completamente la posibilidad de conseguir un escaño parlamentario. Los partidos de menor votación que tienen votos a lo largo del país pueden juntar, como es el caso del Partido Comunista, una votación nacional importante que cualquier sistema proporcional premiaría con algún escaño. El cómputo del voto remanente puede convertirlo también en realidad.

Otro gran defecto del sistema binominal actual es que produce una proporcionalidad dudosa en la representación parlamentaria. Aunque sus defensores insisten que no es cierto que sea tan desproporcional, hay que recordar que la proporcionalidad que hoy vemos es en gran medida 
el producto de la confección de los cupos partidarios en las listas electorales. En todo caso, el cómputo de los votos remanentes permite corregir la proporcionalidad de la representación. No sólo abre la puerta para la representación parlamentaria de las terceras listas, como ya vimos, sino que además corrige, en general, la distribución de las proporciones de los escaños recibidos por los demás partidos. Este efecto puede constatarse en el Cuadro 2, que contiene un resumen completo de los electos por cada partido en la elección de diputados 2005, tanto con y sin el cómputo adicional, mostrando las proporcionalidades de la representación que resultaron y que resultarían con esta reforma.

\section{Cuadro 1}

Distribución por partidos de 15 escaños adicionales según el cómputo de los votos remanentes generados por las elecciones de diputados de 2005

\begin{tabular}{lcccc}
\hline Partidos & $\begin{array}{c}\text { Votos } \\
\text { remanentes }\end{array}$ & $\begin{array}{c}\text { Diputados } \\
\text { elegidos con el } \\
\text { cuociente de Hare }\end{array}$ & $\begin{array}{c}\text { Saldos de } \\
\text { votos remanentes }\end{array}$ & $\begin{array}{c}\text { Diputados } \\
\text { elegidos en 2a } \\
\text { instancia }\end{array}$ \\
\hline UDI & 411.672 & 2 & 128.826 & 1 \\
RN & 327.242 & 2 & 44.396 & - \\
PDC & 648.922 & 4 & 83.230 & 1 \\
PRSD & 19.260 & - & 19.260 & - \\
PPD & 118.381 & - & 118.381 & 1 \\
PS & 151.048 & 1 & 9.625 & - \\
PC & 317.939 & 2 & 35.093 & - \\
PH & 102.314 & - & 102.314 & - \\
I/f/pacto & 24.567 & - & 24.567 & 4 \\
TOTALES & 2.121 .345 & 11 & & \\
\hline
\end{tabular}

Notas: 1. Los saldos de los votos remanentes de los partidos que eligen diputados en base a la división con el cuociente de Hare resultan, primero, de la multiplicación de este cuociente por el número de diputados elegidos, y luego de la resta de este producto del número de votos remanentes inicial. 2. Las siglas son: UDI= Unión Demócrata Independiente; $\mathrm{RN}=$ Renovación Nacional; $\mathrm{PDC}=$ Partido Demócrata Cristiano; $\mathrm{PRSD}=\mathrm{Parti}-$ do Radical Social Demócrata; $\mathrm{PPD}=$ Partido Por la Democracia; PS= Partido Socialista; $\mathrm{PC}=$ Partido Comunista; $\mathrm{PH}=$ Partido Humanista; e I/f / pacto $=$ Independientes fuera de pactos. 3. Para simplificar, el cuadro excluye a los Independientes ligados a las listas A, $\mathrm{B}, \mathrm{C}$ o D, y al PAR, cuya votación es pequeña (véanse el porcentaje de sus votaciones y sus siglas en el Cuadro 2). 


\section{Cuadro 2}

Comparación de la distribución de escaños por partidos en la elección parlamentaria de 2005 sin y con la agregación del cómputo de los votos remanentes

\begin{tabular}{|c|c|c|c|c|c|c|}
\hline $\begin{array}{l}\text { Partidos } \\
\text { o listas }\end{array}$ & $\begin{array}{c}\% \text { de votos } \\
\text { obtenidos } \\
\text { en la elección }\end{array}$ & $\begin{array}{l}\text { Electos } \\
\text { sin el } 2^{\circ} \\
\text { cómputo }\end{array}$ & $\begin{array}{c}\% \text { del total } \\
\text { de electos } \\
\text { sin el } 2^{\circ} \\
\text { cómputo }\end{array}$ & $\begin{array}{c}\text { Electos } \\
\text { con el } \\
\text { voto } \\
\text { remanente }\end{array}$ & $\begin{array}{l}\text { Total final } \\
\text { de electos } \\
\text { con ambos } \\
\text { cómputos }\end{array}$ & $\begin{array}{c}\% \text { del total } \\
\text { final de } \\
\text { electos }\end{array}$ \\
\hline UDI & 22,34 & 33 & 27,5 & 3 & 36 & 26,7 \\
\hline $\mathrm{RN}$ & 14,12 & 19 & 15,8 & 2 & 21 & 15,6 \\
\hline PDC & 20,78 & 20 & 16,7 & 5 & 25 & 18,5 \\
\hline PRSD & 3,51 & 7 & 5,8 & - & 7 & 5,1 \\
\hline PPD & 15,44 & 21 & 17,5 & 1 & 22 & 16,3 \\
\hline PS & 10,02 & 16 & 13,3 & 1 & 17 & 12,6 \\
\hline PC & 5,14 & - & - & 2 & 2 & 1,5 \\
\hline PH & 1,55 & - & - & 1 & 1 & 0,7 \\
\hline PAR & 0,40 & 1 & 0,8 & - & 1 & 0,7 \\
\hline $\mathrm{I} / \mathrm{f} /$ pacto & 0,94 & - & - & - & - & - \\
\hline ILA & 0,46 & - & - & - & - & - \\
\hline ILB & 2,00 & 1 & 0,8 & - & 1 & 0,7 \\
\hline ILC & 0,70 & - & - & - & & - \\
\hline ILD & 2,23 & 2 & 1,7 & - & 2 & 1,5 \\
\hline TOTALES & $99,63^{*}$ & 120 & $99,9^{* *}$ & 15 & 135 & $99,9^{* *}$ \\
\hline
\end{tabular}

${ }^{*}$ Los datos no son los oficiales y están sujetos a pequeñas correcciones que obviamente son necesarias.

** No suma 100 por redondeo al decimal más próximo.

Notas adicionales: Las siglas no contenidas en el Cuadro 1 son: PAR= Partido de Acción Regionalista de Chile; ILA= Independientes Lista A (Fuerza Regional Independiente); ILB $=$ Independientes Lista B (Concertación Democrática); ILC= Independientes Lista C (Juntos Podemos Más); Independientes Lista D (Alianza por Chile).

El efecto correctivo sobre la distribución de los escaños en la Cámara de Diputados que introduce el cómputo de los votos remanentes queda comprobado en el Cuadro 2. Al comparar los porcentajes de la votación nacional con los porcentajes del total de electos sin y con segundo cómputo, queda claro que, en todos los casos, el segundo cómputo los aproxima. La única excepción, sin importancia por lo exigua y por ser producto del aumento del número de elegidos, es la de los independientes asociados a 
los pactos. Este efecto aproximativo en las proporcionalidades se da en las dos direcciones: los partidos que tienen una mayor proporción de electos que de votos reducen el excedente, y los que tienen una menor proporción de elegidos que de sufragios aminoran el déficit. Las desproporciones mayores que permanecen son aquéllas que se producen con los partidos o las listas que no integran los dos pactos mayoritarios o que tienen una relación ambigua con ellos. Por lo mismo, no pueden beneficiarse de los dos cómputos, que obviamente es lo que magnifica la representación. En consecuencia, el cómputo remanente no abre enteramente el dique que excluye a los partidos más pequeños, efecto que viene del número moderado de escaños que se agregan.

Cabe indicar que la distribución de escaños a partir del voto remanente ayuda a corregir además otro gran defecto del sistema binominal: el hecho que genera disparidades desmedidas en el número promedio de votos que requieren los partidos para elegir a sus representantes. Por ejemplo, en la elección de 2005, los diputados elegidos por el PDC recibieron en promedio 64.500 votos, pero sus colegas de la Concertación electos por el PRSD sólo juntaron, en promedio, 32.730, es decir, casi la mitad. Y este efecto no sólo se explica por los electorados más pequeños de los distritos en que el PRSD presentó candidatos. La UDI, en la misma elección, sólo requirió un promedio de 44.134 votos por candidato elegido, a pesar de tener, al igual que el PDC, candidaturas en prácticamente todos los distritos. Estas disparidades varían de una a otra elección a pesar de que se produzcan cambios mínimos en los totales de votos expresados por cada partido. Naturalmente, dada la forma en que se calculan los votos remanentes, los partidos que sufren mayormente las consecuencias de estas disparidades son precisamente los que más acumulan votos en la columna remanente. En consecuencia, resultan ser los primeros premiados con los escaños que así se asignan, como sucedería -ya lo vimos arriba- en beneficio de la Democracia Cristiana en la elección de 2005.

La votación senatorial de 2005 dio 2.309.419 votos remanentes. De éstos, 640.880 provinieron del exceso de votos por sobre el promedio nacional, que fue de 235.755, para elegir a un senador. Estos votos fueron generados por dos senadores electos de la UDI, uno del PPD y una senadora del PDC. Siendo que sólo agregó tres senadores al Senado con cada renovación parcial de éste, el divisor de Hare para esta elección resulta alto, es decir 769.806. Con ello, ni el PDC supera al cuociente, siendo el partido que más votos remanentes obtuvo, con 718.255 , un $47,3 \%$, los cuales vienen del aporte excedentario de la votación de Soledad Alvear. En consecuencia, los tres escaños se asignarían sencillamente a los partidos que tienen las tres primeras mayorías en sus votos remanentes, lo que favorecería al PDC (Andrés Zaldívar en Santiago Poniente); a RN (Lily Pérez de Santiago Oriente); y al PPD (Aníbal Pérez de la novena circunscripción, quien se beneficia principalmente de la alta votación excedentaria de Guido Girardi). La asignación de estos escaños en base al voto remanente mejoraría la proporcionalidad entre la votación recibida y el 
tamaño de la representación elegida en el Senado para la Democracia Cristiana (pasaría de 25\% a 26,1\% de los electos, habiendo conseguido un $29,7 \%$ de la votación). Sucedería lo mismo con el PPD (que aumentaría de un $5 \%$ a un $8,7 \%$ su representación, habiendo recibido un $10,8 \%$ de la votación). Sin embargo, acentuaría la distancia entre el tamaño de la representación y la votación de Renovación Nacional (que pasaría de un $15 \%$ de los veinte electos a un $17,4 \%$ de los mismos con un $10,8 \%$ de la votación). En todo caso, habría que esperar que se cumplan los dos ciclos electorales que producen la renovación completa del Senado para examinar adecuadamente los efectos del cómputo adicional de los votos remanentes. Con solamente tres escaños, el impacto de esta medida es por el momento limitado. Sin embargo, en general va en la dirección esperada.

La segunda ronda de cómputos y asignación de escaños no tiene por qué crear incentivos, a pesar de lo que pudiera temerse, para que los partidos se desprendan de las dos grandes coaliciones pensando que podrán ganar de todas maneras una buena representación parlamentaria por este conducto. Como vimos en estos ejemplos, el cómputo de los votos remanentes distribuye los escaños en forma muy proporcional. Además, no son muchos y, por lo mismo, no convendría basar una estrategia electoral enteramente en ellos, a no ser que el partido en cuestión tenga pocos electores distribuidos a lo largo del país, y sostenga posiciones que lo pongan al margen de las dos coaliciones más grandes. En otras palabras, el segundo cómputo no reduce los incentivos que llevan a la formación de las grandes coaliciones. Permite que partidos pequeños, fuera de las grandes coaliciones, obtengan alguna representación, aunque los principales beneficiados seguirán siendo quienes participan de ellas.

\section{Conclusión}

Esta propuesta muestra que hay una alternativa de reforma del sistema binominal mayoritario por lista, que elimina o aminora sus principales defectos sin requerir su sustitución completa. Esta alternativa tiene también la ventaja, a diferencia de cambiar drásticamente el sistema por uno proporcional, de que no es necesario revisar la distribución de los números de representantes por unidades territoriales de representación. Además, al mantener el aspecto básico de la fórmula binominal, esta reforma altera menos las condiciones bajo las cuales los legisladores actuales fueron elegidos, con lo cual debieran surgir menos reticencias de su parte por considerarla y adoptarla.

De adoptarse la fórmula sugerida aquí, el sistema electoral chileno seguiría siendo único en el mundo. Se mantendría el sistema binominal mayoritario por lista, y los votos remanentes para el segundo cómputo se desprenderían, al menos en lo que se refiere a su segunda fuente, directamente de su operación. Con ello el elector votaría una sola vez, pero de ese voto se derivarían dos cómputos. La noción de hacer dos 
cómputos, uno mayoritario y otro proporcional, no es en sí excepcional. Es una idea que surgió de la democracia alemana de la postguerra. Inicialmente un solo voto servía para ambos cómputos, aunque a partir de 1953 una reforma exigió que cada elector marcase dos preferencias: una por un representante para su distrito y otra por un partido ${ }^{8}$. La confección de este sistema resultó del ánimo de evitar el fraccionamiento partidario extremo de la República de Weimar, medida que tuvo éxito. La fórmula de los dos cómputos ha sido adoptada recientemente con variantes específicas en varios países europeos, incluido Italia.

La ventaja principal de adoptar las sugerencias presentadas aquí es que se mantendrían los incentivos que llevan a la organización del sistema partidario chileno en dos grandes coaliciones, y se evitaría su fraccionamiento. Es más, el primer aspecto de esta reforma -el aumento del número de candidatos- incentivaría la unificación de los partidos más pequeños manteniendo un pluripartidismo moderado. Recordemos que con el sistema d'Hondt usado en Chile después de 1925, se podían presentar más de cien listas "partidarias" distintas en las elecciones, la mayor parte de ellas emparentadas unas con otras por una maraña de pactos. No había cortapisa alguna que previniese que los dirigentes de los partidos que se disputaban entre sí terminasen por organizar sus propias listas de candidatos y vertientes partidarias, produciéndose fácilmente varios partidos de uno solo con calificativos tales como "auténtico", "popular", "unido", "democrático", "tradicional", "obrero", "de Chile", "social cristiano", etcétera, sin que haya faltado nunca la imaginación para acrecentar el repertorio de las etiquetas. Ese excesivo fraccionamiento partidario puede haber estimulado la representatividad democrática al aumentar los matices políticos que se ofrecían al electorado, pero estimulaba un ofertismo de parte de los dirigentes políticos que excedía grandemente las capacidades fiscales del país, y la fragmentación resultante de la representación partidaria en el Congreso dificultaba la gobernabilidad. La posibilidad de crear una nueva etiqueta partidaria y reelegirse de ese modo disminuía la capacidad de los dirigentes de los partidos de imponer su disciplina en las votaciones legislativas. Se pueden diseñar sistemas proporcionales que evitan este tipo de fragmentación extrema, y de hecho una ley electoral de 1962, al prohibir los pactos entre partidos, la redujo en Chile sin abandonar el sistema d'Hondt. Sin embargo, con las reformas sugeridas aquí se puede profundizar la representatividad de la democracia chilena -que sin duda está venida a menos con el sistema electoral actual- sin que se pierda la estructura coalicional que ha caracterizado el sistema de partidos en los últimos años.

La Concertación ha sido la alianza de gobierno más duradera y más exitosa que ha tenido Chile en toda su historia ${ }^{9}$. Representa además la

8. Farrell, pp. 87-88, lo explica bien.

9. Aclaro que la Alianza Liberal de comienzos del siglo XX aún supera en años a la Concertación como etiqueta, pero no gobernó en forma continua y sus partidos integrantes fueron cambiando con el tiempo. 
primera vez -y esto en el marco de la historia política no sólo de Chile sino también de los países católicos de Europa y América- que se juntan durablemente partidos con una agenda social y política parecida pero de vertientes históricas tanto cristianas como seculares (o "laicas" para usar la expresión chilena). No cabe duda que la gobernabilidad ejemplar que el país ha tenido en esta última década y media ha contribuido a su rápido desarrollo, sin ser, por cierto, el único factor en ello. La historia del desarrollo económico chileno registra un largo estancamiento desde 1914, y es sólo a partir de 1990 que el país supera, en términos per cápita expresados en dólares constantes, el nivel que tenía antes de la Primera Guerra Mundial ${ }^{10}$. Además, nunca antes desde 1870 el país había tenido, como ha ocurrido en la última década, una sucesión de años en que la tasa de crecimiento ha superado la tasa de inflación. Paradójicamente, en su intento por favorecer a sus partidarios, la dictadura creó un sistema electoral cuyo resultado inesperado ha sido el de contribuir a la mantención de esta coalición y la de su oposición. El sistema crea déficit democráticos, pero éstos son corregibles -como hemos visto aquí- dejando en pie el aspecto que incentiva fuertemente la estructuración del sistema partidario chileno, tan susceptible al fraccionamiento, en dos grandes coaliciones de partidos afines entre sí.

\section{Bibliografía}

J. Samuel Valenzuela, J. Samuel. Otoño 1995. “Orígenes y transformaciones del sistema de partidos en Chile". Estudios Públicos 58: 5-77.

Farrell, David M. 1998. Comparing Electoral Systems. London: MacMillan.

Valenzuela, J. Samuel. 2006. “Demografía familiar y desarrollo. Chile y Suecia desde 1914". En: J. Samuel Valenzuela, Eugenio Tironi y Timothy R. Scully, C.S.C.. El eslabón perdido: familia, bienestar y modernización en Chile. Santiago: Taurus.

Fecha de recepción: Diciembre de 2005.

Fecha de publicación: Diciembre de 2005.

10. Véase al respecto J. Samuel Valenzuela (2006). 